\title{
Risk Factors for Failure of Nonoperative Treatment for Unilateral Cervical Facet Fractures
}

\author{
Carola Francisca van Eck ${ }^{1}$, Mitchell Stephen Fourman ${ }^{1}$, Amir Mohamad Abtahi ${ }^{1}$, \\ Louis Alarcon', William Fielding Donaldson ${ }^{1}$, Joon Yung Lee ${ }^{1}$ \\ ${ }^{1}$ Department of Orthopaedic Surgery, University of Pittsburgh, Pittsburgh, PA, USA \\ ${ }^{2}$ Department of General and Trauma Surgery, University of Pittsburgh, Pittsburgh, PA, USA
}

\begin{abstract}
Study Design: Retrospective clinical study.
Purpose: The purpose of this study was to determine what percentage of patients who underwent nonoperative management of unilateral non-displaced or minimally displaced facet fractures progressed radiographically and to determine what percentage of patients required surgical intervention and to identify risk factors for failure of conservative management.

Overview of Literature: According to most commonly used classification systems, unilateral, non-and minimally displaced facet fractures are be amendable to nonoperative management.

Methods: A retrospective review of the Trauma Registry of a Level I trauma center was performed to identify all patients diagnosed with a non- or minimally displaced unilateral facet fracture which was managed nonoperatively. Several demographic variables and clinical outcomes were recorded. Using computed tomography scanning and plain radiographs, fracture pattern, listhesis, displacement, angle and percentage of the facet that included the fracture were determined. Radiographic progression was defined as the occurrence of listhesis of more than $10 \%$ of the anterior-posterior dimensions of the inferior vertebral body during radiographic follow-up. Failure of conservative management was defined as a patient requiring surgical intervention after initially being managed nonoperatively.

Results: Seventy-four patients were included. Fifteen patients (20\%) progressed radiographically. However, only 2 developed radicular symptoms and none developed myelopathy or other catastrophic cord related symptoms. Seven patients (9\%) underwent surgery. Indications for surgery included significant radiographic progression and/or radicular symptoms. Risk factors for failure of conservative management included presence of radiculopathy at the time of presentation, a higher body mass index, increased Injury Severity Score, greater initial fracture displacement and more than $2 \mathrm{~mm}$ of listhesis.

Conclusions: Patients with non-displaced or minimally displaced facet fractures who do not have neurological symptoms at the time of presentation can safely be managed conservatively with careful observation and follow-up.
\end{abstract}

Keywords: Cervical spine; Facet fracture; Non-displaced; Nonoperative treatment; Failure rate

\section{Introduction}

Subaxial cervical spine fractures are commonly encoun- tered in clinical practice. Although many cervical facet fractures are associated with significant displacement and instability, approximately $5 \%$ of all traumatic cervical

\footnotetext{
Received Sep 19, 2016; Revised Oct 21, 2016; Accepted Oct 31, 2016

Corresponding author: Carola Francisca van Eck

Department of Orthopaedic Surgery, University of Pittsburgh,

Kaufman Building Suite 1011, 3471 Fifth Avenue, Pittsburgh, PA 15213, USA

Tel: +1-412-605-3265, Fax: +1-412-687-0802, E-mail: vaneckcf@upmc.edu
} 
spine injuries involve isolated non-displaced or minimally displaced facet fractures [1]. The facet joints facilitate cervical range of motion while preventing significant translation and rotation and are the main stabilizers of the cervical spine.

A number of subaxial spine injury classification systems have been proposed, most recently the Subaxial Cervical Spine Injury Classification System [2-4]. The goal of these classification systems is to facilitate decision making when treating such injuries. Particularly with regards to operative versus nonoperative management [5-10]. According to most of the commonly used classification systems, unilateral, non-displaced and minimally displaced facet fractures without any sign of a spinal cord injury would be amendable to nonoperative management. However, no consensus exists with regards to the optimal management of these injuries. In addition, the failure rate and predictors of failure after conservative management remain unknown [11]. Most studies on this topic have used a study design were the outcomes of nonoperative treatment were compared to operative treatment This leads to a high degree of selection bias [11].

The primary aim of this study was to determine what percentage of patients who underwent nonoperative management of unilateral non- or minimally displaced facet fractures progressed radiographically as determined by the occurrence of listhesis during follow-up. Furthermore, this study aimed to determine what percentage of patients required surgical intervention and to identify risk factors for failure of conservative management.

\section{Materials and Methods}

Prior to the start of this study, Institutional Review Board (IRB) approval was obtained (PRO15030378).

At our institution a Trauma Registry is kept by the General and Trauma Surgery Department, in which all patients presenting as a trauma to our level one trauma center are included (IRB no. PRO07090338). This registry was searched for all patients documented to have a cervical spine fracture, treated by the Orthopaedic Surgery department between 2002 and 2014. From these patients, the chart was reviewed to include only those patients diagnosed with a non-displaced or minimally displaced unilateral facet fracture which was initially managed nonoperatively. Patients were excluded if they underwent cervical spine surgery during their index admission. Other exclusion criteria included: concomitant additional cervical spine fractures, severe underlying disease patterns precluding accurate radiographic analysis, and evidence of spinal cord injury.

A retrospective chart review of these patients was performed to record the following demographic variables: age, gender, height, weight, body mass index (BMI), location of fracture, mechanism of injury, American Spinal Injury Association (ASIA) classification, injury severity score (ISS), Glasgow Coma Scale (GCS), associated injuries, smoking status, comorbidities, Charlson comorbidity index, symptoms on admission (neck pain, radiculopathy, myelopathy) and nonoperative treatment rendered.

The following outcomes were recorded: discharge location, whether they underwent surgery at any time during
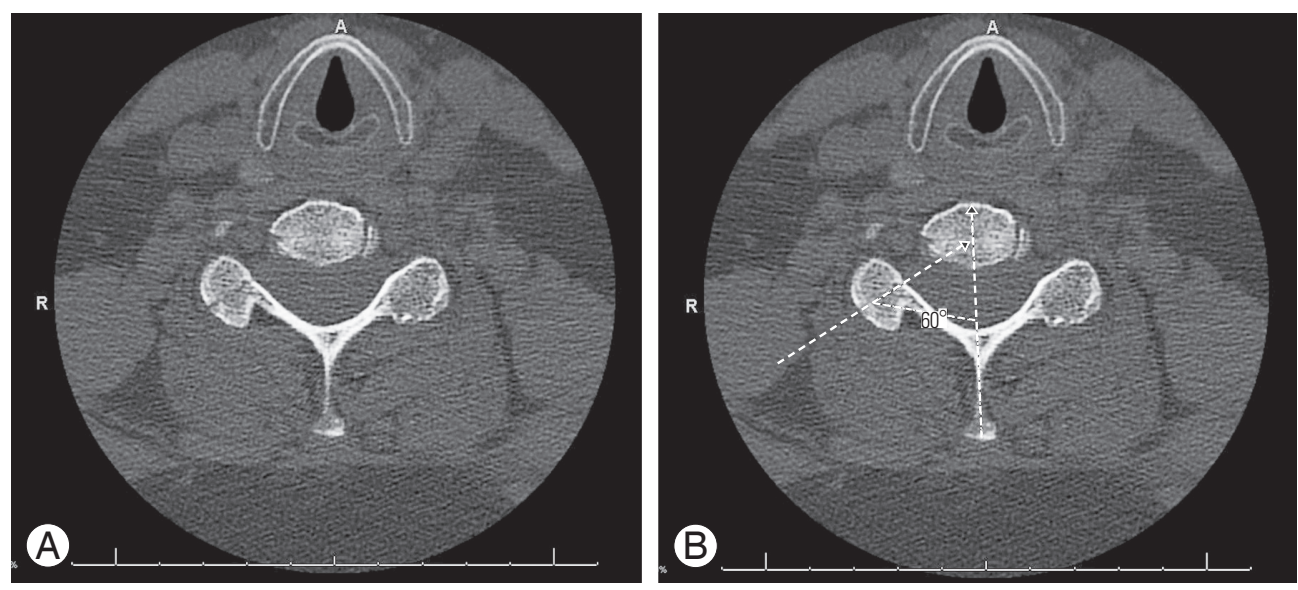

Fig. 1. (A) Axial computed tomography (CT) scan image showing a unilateral facet fracture. (B) Angle of the fracture line as measured on the axial CT image, relative to the sagittal plane. 
follow-up, symptoms during all clinical visits (neck pain, radiculopathy, myelopathy and catastrophic cord related symptoms such as paraplegia or tetraplegia) and length of follow-up. Using computed tomography scanning (CT) and plain radiographs from admission and follow-up, a spine fellowship training orthopaedic surgeon recorded: fracture pattern, listhesis, fracture displacement, the angle of the fracture line on axial CT relative to the sagittal plane (Fig. 1) and the one and two dimensional measurements of the facet and the fracture (Fig. 2). Based on these last measurements, the percentage of the facet that included the fracture was calculated. If patients did undergo surgery, the following additional outcomes were recorded: complications, non-union, re-operation rate.

Radiographic progression was defined as the occurrence of listhesis of more than $10 \%$ of the anteriorposterior dimensions of the inferior vertebral body during radiographic follow-up. This was measured either on flexion-extension radiographs or a CT scan of the cervical spine performed during follow-up. Failure of conservative management was defined as patients requiring surgical intervention after initially being managed nonoperatively.

\section{Statistical analysis}

Descriptive statistics including mean, range and standard deviation were calculated for all continuous variables. Ratios and percentages were calculated for the categorical variables. For the continuous variables, an independent $t$ test was used to determine differences between those subjects who failed nonoperative management and those subjects who were successfully treated conservatively. A subsequent regression analysis was used to combine the found risk factors into a single correlation coefficient. For the categorical variables, the chi-squared test was used to determine differences between those subjects who failed nonoperative management and those subjects who were successfully treated conservatively. Odds ratio was then reported for the significant risk factors. Alpha level of statistical significance was set at 0.05 . All analyses were performed using SPSS ver. 20 (IBM Corp., Armonk, NY, USA).

\section{Results}

The trauma registry included 3,099 patients who were evaluated between 2002 and 2014 and who had a cervical spine fracture. After detailed chart review of these patients, 103 patients were identified with a unilateral non-displaced or minimally displaced facet fracture who were initially managed nonoperatively. After evaluation of all available imaging, 29 patients were excluded due to the presence of additional concomitant cervical spine fractures confounding treatment strategy or outcome, or severe underlying disease patterns precluding accurate radiographic analysis. This left 74 patients available for final inclusion.

The average age was $45 \pm 17$ years (range, $17-87$ years)
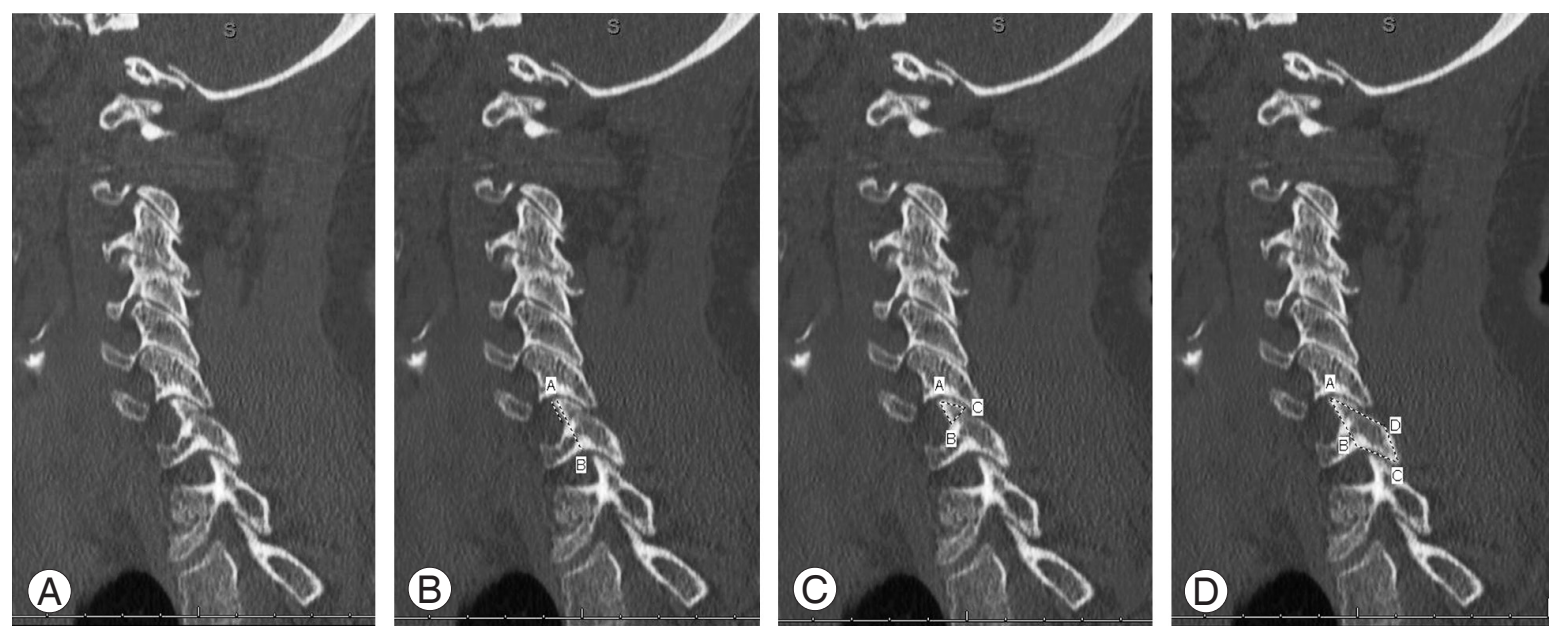

Fig. 2. (A) Sagittal computed tomography (CT) image showing a unilateral facet fracture. (B) Measurement of the height of the fracture (line A) and height of the facet (line B) used to calculate the 2-dimensional percentage of the facet that includes the fracture $(A / B \times$ 100\%). (C) Measurement of the area of the fracture and (D) measurement of the area of the facet used the calculate the 3-dimensional percentage of the facet that includes the fracture $[(A \times B \times C) /(A \times B \times C \times D) \times 100 \%]$. 
and BMI was $28 \pm 6$ (range, 17-46). Twenty-one patients $(28 \%)$ were female and $26(25 \%)$ were smokers. In the majority of patients, a motor vehicle accident was the mechanism of injury (41 patients, 55\%). Other mechanisms included: 15 falls (20\%), 6 motor cycle accidents (8\%), 3 assaults (4\%), 2 pedestrian versus car (3\%), 2 sport/recreational activities (3\%), 2 bicycle accidents (3\%), and 2 all-terrain vehicle accidents (3\%). All patients were neurologically intact (ASIA-E) upon arrival. Five patients (7\%) had radicular symptoms on initial presentation, all patients (100\%) complained of neck pain. Forty-one patients (55\%) were polytrauma patients. Twenty patients (27\%) had associated musculoskeletal injuries, 3 (4\%) abdominal injuries, 7 (10\%) had soft tissue trauma, 5 (7\%) had chest trauma, and 9 (12\%) facial trauma. Average ISS was 13 (range, 4-43) and GCS 14 (range, 3-15). Average Charlston comorbidity score was 0.7 (range, $0-5$ ).

The characteristics of the fracture as determined on the CT scan from admission are displayed in Table 1.

All patients were treated in a hard cervical collar. No patients suffered any morbidity associated with wearing the collar. Average length of stay for the initial admission was 4 days (range, 1-25 days). Average intensive care unit length of stay was 1 day (range, 0-25 days). Sixty-three patients (85\%) were discharged home from the hospital, $8(11 \%)$ were discharge to a skilled nursing facility and 3 patients (4\%) went to a rehabilitation facility.
Table 1. Facet fracture characteristics

\begin{tabular}{|c|c|}
\hline Variable & Value \\
\hline \multicolumn{2}{|l|}{ Side } \\
\hline Right & $38(52)$ \\
\hline Left & $36(49)$ \\
\hline \multicolumn{2}{|l|}{ Location } \\
\hline Superior facet & $59(80)$ \\
\hline Inferior facet & $15(20)$ \\
\hline \multicolumn{2}{|l|}{ Cervical level } \\
\hline C3 & $7(10)$ \\
\hline C4 & $2(3)$ \\
\hline C5 & $8(11)$ \\
\hline C6 & $23(31)$ \\
\hline C7 & $34(46)$ \\
\hline \multicolumn{2}{|l|}{ Fracture pattern } \\
\hline Simple & $55(74)$ \\
\hline Comminuted & $19(26)$ \\
\hline \multicolumn{2}{|l|}{ Listhesis } \\
\hline$<2 \mathrm{~mm}$ & $70(95)$ \\
\hline$>2 \mathrm{~mm}$ & $4(5)$ \\
\hline Displacement (mm) & $1.1 \pm 1.7$ \\
\hline Fracture angle $\left({ }^{\circ}\right)$ & $48 \pm 20$ \\
\hline \% Facet involved in fracture (mm) & $74 \pm 24$ \\
\hline$\%$ Facet involved in fracture $\left(\mathrm{mm}^{2}\right)$ & $1.8 \pm 1.3$ \\
\hline
\end{tabular}

Values are presented as number (\%) or mean \pm standard deviation.

Table 2. Risk factors for radiographic progression (continuous variables)

\begin{tabular}{lccc} 
Variable & $\begin{array}{c}\text { Radiographic progression } \\
(\mathrm{n}=15)\end{array}$ & $\begin{array}{c}\text { No radiographic } \\
\text { progression }(\mathrm{n}=43)\end{array}$ & $p$-value \\
\hline Age & $48 \pm 16$ & $44 \pm 17$ & 0.384 \\
\hline Height & $173 \pm 12$ & $172 \pm 11$ & 0.703 \\
\hline Weight & $95 \pm 22$ & $77 \pm 14$ & $0.002^{2)}$ \\
\hline BMI & $31 \pm 6$ & $26 \pm 5$ & $0.003^{\text {a) }}$ \\
\hline ISS & $11 \pm 8$ & $15 \pm 11$ & 0.188 \\
\hline GCS & $15 \pm 0$ & $14 \pm 3$ & 0.198 \\
\hline Charleston comorbidity index & $1.3 \pm 1.8$ & $0.5 \pm 0.8$ & $0.022^{2)}$ \\
\hline Fracture displacement & $2.3 \pm 2.3$ & $0.9 \pm 1.5$ & $0.010^{0^{a}}$ \\
\hline Fracture angle & $54 \pm 16$ & $50 \pm 20$ & 0.717 \\
\hline \% Facet involved in fracture $\left(\mathrm{mm}^{2}\right)$ & $84 \pm 19$ & $74 \pm 22$ & $0.050^{\text {a) }}$ \\
\hline \% Facet involved in fracture $\left(\mathrm{mm}^{2}\right)$ & $2.2 \pm 1.4$ & $1.8 \pm 1.2$ & 0.294 \\
\hline
\end{tabular}

Values are presented as mean \pm standard deviation.

BMI, body mass index; ISS, injury severity score; GCS, Glasgow Coma Scale.

a) Significant difference. 
Table 3. Risk factors for radiographic progression (nominal variables)

\begin{tabular}{|c|c|c|c|}
\hline Variable & $\begin{array}{l}\text { Radiographic progression } \\
\qquad(\mathrm{n}=15)\end{array}$ & $\begin{array}{l}\text { No radiographic } \\
\text { progression }(n=43)\end{array}$ & $p$-value \\
\hline Gender male & $9(60)$ & $28(65)$ & 0.723 \\
\hline Side right & $6(40)$ & $26(60)$ & 0.170 \\
\hline Location superior & $12(80)$ & $35(81)$ & 0.906 \\
\hline Cervical level & & & 0.885 \\
\hline C3 & $1(7)$ & $3(7)$ & \\
\hline $\mathrm{C} 4$ & $1(7)$ & $1(2)$ & \\
\hline $\mathrm{C5}$ & $2(13)$ & $4(9)$ & \\
\hline C6 & $4(27)$ & $16(37)$ & \\
\hline $\mathrm{C7}$ & $7(47)$ & $19(44)$ & \\
\hline Smoking yes & $3(20)$ & $15(35)$ & 0.283 \\
\hline Radiculopathy on admission & $3(20)$ & $2(5)$ & 0.073 \\
\hline Myelopathy on admission & 0 & 0 & 1.000 \\
\hline Neck pain on admission & $15(10)$ & $43(100)$ & 1.000 \\
\hline Fracture pattern comminuted & $5(33)$ & $9(21)$ & 0.334 \\
\hline Listhesis >2 mm admission CT & $2(13)$ & $1(2)$ & 0.097 \\
\hline
\end{tabular}

Values are presented as number (\%).

CT, computed tomography.

Fifteen patients $(20 \%)$ progressed radiographically and developed listhesis during follow-up. However, only 2 of these patients developed radicular symptoms during follow-up. No patients were found to have new onset myelopathy or catastrophic cord related symptoms such as paraplegia or tetraplegia during follow-up. Sixteen patients (22\%) did not have adequate radiographic followup. Risk factors for radiographic progression are displayed in Table 2 for the continuous variables and Table 3 for the categorical variables. None of the categorical variables proved significant risk factors. For the continuous variables weight, BMI, Charlston comorbidity score, fracture displacement and the percentage of the facet involved in the fracture showed significance with the $t$-test. Regression analysis revealed that of these had a combined pseudo r-squared of 0.48 , indicating they account for almost half of the radiographic progression risk.

Eight patients underwent surgery during the follow-up period. However, one of these patients did not have surgery until 7 years after the initial trauma. Therefore only 7 patients (9\%) were considered to be have failed conservative management. For these patients, time between injury and surgery ranged from 2 to 10 weeks. Indications for surgery included significant radiographic progres- sion and/or radicular symptoms. Procedures included 1 laminectomy (12.5\%), 2 anterior cervical discectomy and fusions (ACDF) with allograft (25\%), 3 ACDF with autograft $(37.5 \%)$ and 2 posterior spinal fusions (25\%). One patient underwent a second surgery during follow-up several years after the index procedure for adjacent segment degeneration leading to cervical spinal stenosis. Risk factors for failure of conservative management are displayed in Table 4 for the continuous variables and Table 5 for the categorical variables. Of the categorical variables, radiculopathy on admission and listhesis of more than 2 $\mathrm{mm}$ on initial CT scan proved significant risk factors with odds ratios of 6.9 and 10.7, respectively. For the continuous variables BMI, ISS and fracture displacement showed significance with the $t$-test. Regression analysis revealed that of these had a combined pseudo r-squared of 0.44 , indicating they account for $44 \%$ of the risk for failing conservative management.

For the 8 patients who underwent surgery, no cases of dysphagia, wound complications, infections, deep venous thrombosis, pulmonary embolism, pneumonia, iatrogenic nerve or spinal cord injury, dural tears or non-unions were recorded. There was one re-operation (12.5\%) but this was several years after the index procedure. 
Table 4. Risk factors for failure of conservative management (continuous variables)

\begin{tabular}{lccc} 
Variable & Failure $(\mathrm{n}=8)$ & Success $(\mathrm{n}=66)$ & $p$-value \\
Age & $47 \pm 15$ & $44 \pm 17$ & 0.743 \\
Height & $171 \pm 10$ & $173 \pm 11$ & 0.767 \\
\hline Weight & $93 \pm 20$ & $81 \pm 17$ & 0.070 \\
BMI & $32 \pm 6$ & $27 \pm 6$ & $0.042^{\text {a) }}$ \\
\hline ISS & $20 \pm 11$ & $13 \pm 9$ & $0.038^{\text {a) }}$ \\
GCS & $13 \pm 5$ & $14 \pm 2$ & 0.297 \\
Charlston comorbidity index & $0.6 \pm 0.7$ & $0.7 \pm 1.2$ & 0.815 \\
Fracture displacement & $3.3 \pm 2.7$ & $1.9 \pm 1.5$ & $<0.001^{\text {a) }}$ \\
\hline Fracture angle & $49 \pm 19$ & $48 \pm 21$ & 0.833 \\
\hline$\%$ Facet involved in fracture $\left(\mathrm{mm}^{2}\right.$ & $83 \pm 18$ & $73 \pm 24$ & 0.787 \\
\hline Facet involved in fracture $\left(\mathrm{mm}^{2}\right)$ & $2.0 \pm 1.3$ & $1.8 \pm 1.3$ & 0.245 \\
\hline
\end{tabular}

Values are presented as mean \pm standard deviation.

BMI, body mass index; ISS, injury severity score; GCS, Glasgow Coma Scale.

a) Significant difference.

Table 5. Risk factors for failure of conservative management (nominal variables)

\begin{tabular}{|c|c|c|c|}
\hline Variable & Failure $(n=8)$ & Success $(n=66)$ & $p$-value \\
\hline Gender male & $6(75)$ & $47(71)$ & 0.822 \\
\hline Side right & $2(25)$ & $36(55)$ & 0.114 \\
\hline Location superior & $6(75)$ & $53(80)$ & 0.725 \\
\hline Cervical level & & & 0.457 \\
\hline C3 & $2(25)$ & $5(8)$ & \\
\hline C4 & $0(0)$ & $2(3)$ & \\
\hline C5 & $0(0)$ & $8(12)$ & \\
\hline C6 & $2(25)$ & $21(32)$ & \\
\hline C7 & $4(50)$ & $30(45)$ & \\
\hline Smoking & $2(25)$ & $24(36)$ & 0.525 \\
\hline Radiculopathy on admission & $2(25)$ & $3(5)$ & $0.031^{\mathrm{a})}(0 \mathrm{R} 6.9)$ \\
\hline Myelopathy on admission & $0(0)$ & $1(2)$ & 0.724 \\
\hline Neck pain on admission & $8(100)$ & $66(100)$ & 1.000 \\
\hline Fracture pattern comminuted & $4(50)$ & $15(23)$ & 0.095 \\
\hline Listhesis >2 mm & $2(25)$ & $2(3)$ & $0.009^{\mathrm{a})}(\mathrm{OR} 10.7)$ \\
\hline
\end{tabular}

Values are presented as number (\%).

$\mathrm{OR}$, odds ratio.

a) Significant difference.

\section{Discussion}

The most important finding of this study is that patients with non-displaced or minimally displaced facet fractures who do not have neurological symptoms at the time of presentation can safely be managed conservatively. The failure rate with conservative management was $9 \%$. Risk factors for failure included the presence of radiculopathy at the time of presentation, a higher BMI, increased ISS, greater initial fracture displacement and more than $2 \mathrm{~mm}$ of listhesis on the initial post-injury CT scan. Twenty percent of patient progressed radiographically during 
follow-up, as determined by the occurrence of more than $10 \%$ of listhesis during follow-up. However, only 2 of these patients developed radicular symptoms during follow-up and none developed new onset myelopathy or catastrophic cord related symptoms such as paraplegia or tetraplegia during follow-up. Risk factors for radiographic progression included a higher body weight, increased BMI, a higher Charlston comorbidity score, greater initial fracture displacement, and greater percentage of the facet height involved in the fracture. In addition, none of the patients managed conservatively in this study developed complications related to immobilization in a cervical collar, as has been a concern highlighted in previous studies [12-15]. This suggests that careful observation and follow up in patients with unilateral non-displaced or minimally displaced facet fractures without any neurological symptoms is acceptable and may be successful.

The failure rate of nonoperative treated fractures in the present study was significantly lower than that reported by Aarabi et al. [16] who found a $60 \%$ failure rate in their cohort. Their study found that a younger age was associated with a higher chance of treatment failure. However, when they compared surgical and non-surgical treatment, age was a risk factor for treatment failure in both arms of the study [16]. Beyer et al. [1] also reported a higher failure rate of nonoperative treatment in their study $(25 \%)$ then in the present study. However, they also included fracture dislocations and facet dislocations. Furthermore, nonoperative treatment in their study consisted of a halo. Dvorak et al. [17] compared surgically and non-surgically managed patients and found a lower SF-36 score and higher bodily pain score in patients managed nonoperatively. However, their study included patients with all types of facet injuries including unilateral facet fractures, subluxations and dislocations. Spector et al. [18] reported a $21 \%$ failure rate of nonoperative management of unilateral facet fractures in their cohort. They found that height of the fracture fragment, articular fracture height and height of the fracture fragment expressed as a percentage of the contralateral intact lateral mass were correlated with treatment failure [18]. They recommended that patients with unilateral cervical facet fractures involving more than $40 \%$ of the absolute height of the intact lateral mass or an absolute height of more than $1 \mathrm{~cm}$ are at increased risk for failure of nonoperative treatment [18]. Similarly, in the present study we found that a greater percentage of the facet height involved in the fracture was predictive of radiographic progression. However, in contrast to previous studies, we found that the only radiographic factors predictive of clinical failure were the initial fracture displacement and listhesis greater than $2 \mathrm{~mm}$ on initial imaging.

Kepler et al. [19] performed a systematic review of literature on cervical facet fractures and included 11 studies with 368 patients. They concluded that in comparison with nonoperative treatments, operative treatments provided a more successful outcome in terms of failure of treatment to maintain reduction for patients with cervical facet fractures. Operative treatment appeared to provide superior results to the nonoperative treatments assessed [19]. However, again all types of facet fracture were included in this review, including displaced ones and those associated with dislocations or subluxation.

Based on a purely radiographic analysis, Halliday et al. [20] recommended to decide for surgical treatment based on presence of subluxation and the integrity of the ligamentous structures, which was something which was not assessed in the present study as not all patients underwent magnetic resonance imaging (MRI). Several other authors have advocated for MRI as well to guide treatment of facet injuries [21]. Since the present study was conducted in a level I trauma center, CT scan was the mainstay in making the diagnosis of a cervical spine injury. MRI was only performed if deemed indicated by the orthopaedic surgeon.

The failure rate of conservative management of unilateral non-displaced or minimally displaced facet fractures reported in prior studies varies, which might be due to different protocols amongst institutions and differences in comfort level of the providers in managing these injuries nonoperatively. In addition, there is a difference between radiographic progression and true failure of nonoperative management. Future studies will necessary to determine the long term outcome of patients with radiographic progression, which was not assessed in the present study. However, only two of these patients (10\%) in the present study developed radicular symptoms and there were no catastrophic neurological complications such as myelopathy, paraplegia and tetraplegia. Other limitations of this study are that it was a retrospective chart review study. Radiographic follow-up was insufficient for $22 \%$ of the included patients. Average follow-up was 9 months, as by this time most patients were discharged from follow-up as their fracture had united. 


\section{Conclusions}

In conclusion, the results of this study indicate that patients with non-displaced or minimally displaced facet fractures who do not have neurological symptoms at the time of presentation can safely be managed conservatively with careful observation and follow up.

\section{Conflict of Interest}

No potential conflict of interest relevant to this article was reported.

\section{ORCID}

Carola Francisca van Eck: 0000-0002-6657-0001

\section{References}

1. Beyer CA, Cabanela ME, Berquist TH. Unilateral facet dislocations and fracture-dislocations of the cervical spine. J Bone Joint Surg Br 1991;73:977-81.

2. Song KJ, Lee SK, Ham DH, Kim YJ, Choi BW. Limitation of previous Allen classification and subaxial cervical spine injury classification (SLIC) system in distractive-extension injury of cervical spine: proposal of modified classification system. Eur Spine J 2016;25:74-9.

3. de la Rua Julio R, Claudio CG, Tomas VP. The surgical approach to subaxial cervical spine injuries: an evidence-based algorithm based on the SLIC classification system. Spine (Phila Pa 1976) 2008;33:2124.

4. Dvorak MF, Fisher CG, Fehlings MG, et al. The surgical approach to subaxial cervical spine injuries: an evidence-based algorithm based on the SLIC classification system. Spine (Phila Pa 1976) 2007;32:2620-9.

5. Allen BL Jr, Ferguson RL, Lehmann TR, O’Brien RP. A mechanistic classification of closed, indirect fractures and dislocations of the lower cervical spine. Spine (Phila Pa 1976) 1982;7:1-27.

6. Anderson PA, Moore TA, Davis KW, et al. Cervical spine injury severity score: assessment of reliability. J Bone Joint Surg Am 2007;89:1057-65.

7. Harris JH Jr, Edeiken-Monroe B, Kopaniky DR. A practical classification of acute cervical spine injuries. Orthop Clin North Am 1986;17:15-30.

8. Vaccaro AR, Hulbert RJ, Patel AA, et al. The subaxial cervical spine injury classification system: a novel approach to recognize the importance of morphology, neurology, and integrity of the disco-ligamentous complex. Spine (Phila Pa 1976) 2007;32:2365-74.

9. Lee SH, Sung JK. Unilateral lateral mass-facet fractures with rotational instability: new classification and a review of 39 cases treated conservatively and with single segment anterior fusion. J Trauma 2009;66:758-67.

10. Rabb CH, Lopez J, Beauchamp K, Witt P, Bolles G, Dwyer A. Unilateral cervical facet fractures with subluxation: injury patterns and treatment. J Spinal Disord Tech 2007;20:416-22.

11. Dvorak M, Vaccaro AR, Hermsmeyer J, Norvell DC. Unilateral facet dislocations: is surgery really the preferred option? Evid Based Spine Care J 2010;1:57-65.

12. Delcourt T, Begue T, Saintyves G, Mebtouche N, Cottin P. Management of upper cervical spine fractures in elderly patients: current trends and outcomes. Injury 2015;46 Suppl 1:S24-7.

13. Ham HW, Schoonhoven LL, Galer AA, ShortridgeBaggett LL. Cervical collar-related pressure ulcers in trauma patients in intensive care unit. J Trauma Nurs 2014;21:94-102.

14. Molinari WJ 3rd, Molinari RW, Khera OA, Gruhn WL. Functional outcomes, morbidity, mortality, and fracture healing in 58 consecutive patients with geriatric odontoid fracture treated with cervical collar or posterior fusion. Global Spine J 2013;3:21-32.

15. Moran C, Kipen E, Chan P, et al. Understanding post-hospital morbidity associated with immobilisation of cervical spine fractures in older people using geriatric medicine assessment techniques: a pilot study. Injury 2013;44:1838-42.

16. Aarabi B, Mirvis S, Shanmuganathan K, et al. Comparative effectiveness of surgical versus nonoperative management of unilateral, nondisplaced, subaxial cervical spine facet fractures without evidence of spinal cord injury: clinical article. J Neurosurg Spine 2014;20:270-7.

17. Dvorak MF, Fisher CG, Aarabi B, et al. Clinical outcomes of 90 isolated unilateral facet fractures, subluxations, and dislocations treated surgically and nonoperatively. Spine (Phila Pa 1976) 2007;32:300713.

18. Spector LR, Kim DH, Affonso J, Albert TJ, Hilibrand AS, Vaccaro AR. Use of computed tomography to 
predict failure of nonoperative treatment of unilateral facet fractures of the cervical spine. Spine (Phila Pa 1976) 2006;31:2827-35.

19. Kepler CK, Vaccaro AR, Chen E, et al. Treatment of isolated cervical facet fractures: a systematic review. J Neurosurg Spine 2016;24:347-54.

20. Halliday AL, Henderson BR, Hart BL, Benzel EC. The management of unilateral lateral mass/facet fractures of the subaxial cervical spine: the use of magnetic resonance imaging to predict instability. Spine (Phila Pa 1976) 1997;22:2614-21.

21. Hart RA. Cervical facet dislocation: when is magnetic resonance imaging indicated? Spine (Phila Pa 1976) 2002;27:116-7. 